\title{
Fusion proteins and fusion pores
}

\author{
Workshop: Regulated exocytosis and the vesicle cycle
}

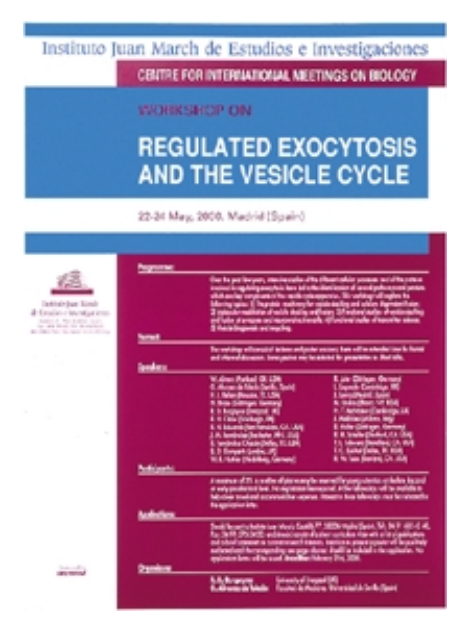

Sponsored by the Juan March Foundation and organized by R.D. Burgoyne and G. Alvarez de Toledo, Madrid, Spain, May 22-24, 2000 .

\section{Introduction}

A meeting was recently held at the Juan March Foundation in Madrid (22-24 May, 2000) on the topic 'Regulated exocytosis and the vesicle cycle'. This small discussion meeting brought together speakers using molecular, electrophysiological or a combination of approaches in the study of exocytosis and vesicle retrieval. It was clear from the presentations and discussions that there is now reasonable agreement about the key proteins involved in exocytosis, but questions were raised about the roles of other proteins that had previously been implicated in this process but may have other functions. The other major area of discussion related to the existence of a rapidly reversible fusion pore, and the significance of, and possible mechanisms for, 'kiss-and-run' fusion. Here we highlight some of the emerging ideas and issues that generated discussion at the meeting.

\section{The proteins}

The most generally expressed view was that the so-called SNARE complex, comprising syntaxin, SNAP-25 and VAMP, is likely to be the core machinery that drives membrane fusion. Indeed, it was noted that the image of the SNARE complex structure, solved by the groups of Axel Brunger and Reinhard Jahn (Sutton et al., 1998), almost became the logo of the meeting. One controversial aspect of the SNARE complex, however, has been whether the pairing of a vesicle SNARE with a target membrane SNARE could contribute specificity to vesicle docking. Recently, other mechanisms for vesicle docking have emerged, and SNARE proteins in vitro show highly promiscuous interactions with one another, suggesting that this may not be the case. Richard Scheller (Stanford, CA), however, presented data from permeabilized PC12 cells that revealed a much higher specificity of SNARE interactions in vivo. The assembly of the SNARE complex into a four-helix bundle involves interactions between the coiled domains of the SNARE proteins. Scheller's lab was able to repair a defect due to botulinum neurotoxin cleavage of SNAP-25 by introducing recombinant coils from SNAP-25. Furthermore, the introduction of specific VAMP or syntaxin coils served to competitively inhibit exocytosis. These studies demonstrated that coils from different SNARE family members are capable of conferring specificity to SNARE interactions, suggesting that specific SNARE pairing may contribute to the overall control of vesicle docking (Scales et al., 2000).

How could SNARE complex formation drive membrane fusion? This aspect was discussed by several speakers including Richard Scheller, Reinhard Jahn (Göttingen, Germany) and Erwin Neher (Göttingen, Germany). Despite minor differences between models, the key idea that has gained acceptance is that the SNAREs form an initial 'loose' complex, and that it is the final zippering into the 'tight' complex that provides the energy to pull membranes into the fused state. According to this model, the structure of the SNARE complex as resolved by Sutton et al. (1998) represents the endpoint of fusion, a state that is then reversed by the disassembling effects of $\alpha$-SNAP and NSF.

In addition to the SNAREs, many other proteins have been implicated in exocytosis. For $\mathrm{Ca}^{2+}$-activated exocytosis, a $\mathrm{Ca}^{2+}$ receptor is required, of course, and the most frequently discussed candidate was synaptotagmin. While it is clear that synaptotagmin plays a crucial role in exocytosis, there is still debate over whether it is the $\mathrm{Ca}^{2+}-$ receptor. This question has been addressed by genetic approaches. Tom Schwarz (Boston, $\mathrm{MA}$ ) described data from Drosophila mutants in which synaptotagmins with specific $\mathrm{C}_{2} \mathrm{~A}$ domain mutations that disrupt $\mathrm{Ca}^{2+}$ dependent interactions were expressed in a null background. These forms of synaptotagmin preserved viability of the flies and restored neurotransmitter release, arguing against a crucial $\mathrm{Ca}^{2+}-$ receptor function for synaptotagmin. A related approach was discussed by Rafael Fernandez-Chacon (Dallas, TX). He described the introduction of mutated forms of synaptotagmin into mice. The phenotype of these mice has yet to be fully 


\section{meeting report}

characterized, and the exact function of synaptotagmin will remain an important issue for some time to come.

Other proteins that play key, but undefined, roles in the control of exocytosis include munc-18/nSec 1 and cysteine string protein (Csp). Munc-18 forms a tight complex with syntaxin, as described by both Scheller and Tom Südhof (Dallas, TX). Südhof discussed data from a knock-out mouse that demonstrated its essential requirement for neurotransmitter release. Important issues that need to be resolved include the questions of how and when the munc-18-syntaxin complex is pulled apart to allow syntaxin to participate in SNARE complex assembly, and whether munc-18 has any additional functions in late stages of exocytosis.

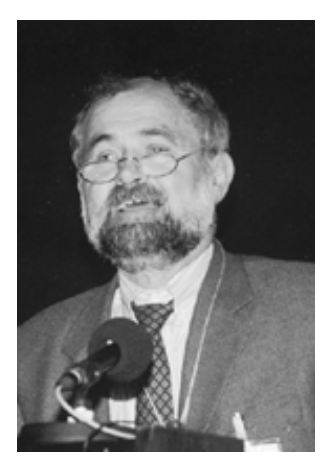

Csp is a chaperone-like protein that has been implicated in late stages of exocytosis. These conclusions are based on an analysis of the effects of its overexpression on the kinetics of individual release events using carbon-fibre amperometry in chromaffin cells (Bob Burgoyne, Liverpool, UK). A Csp knockout mouse has been generated, and it is initially viable but dies within $\sim 5$ weeks after birth (Fernandez-Chacon). Further analysis of these mice will hopefully provide additional clues as to the function of Csp. Also characterized by knock-out analysis in mice were the

Erwin Neher

functions of munc-13 (Nils Brose, Göttingen, Germany), which appears to be important for the priming of synaptic vesicles for release. Also, Erwin Neher presented data showing a stimulatory effect of munc-13 on the priming of dense-core granules in adrenal chromaffin cells, indicating that this may be a general function of munc-13.

The proteins discussed above were originally identified as components of the exocytosis machinery through biochemical approaches. Genetic studies confirmed that they play key roles. In contrast, other players in the process of exocytosis are beginning to fall out of the picture as a result of more rigorous functional analyses. Two of these are the VAMP-binding protein VAP-33 and the SNAP-25 binding protein HRS. Analysis of null mutants in Drosophila (Hugo Bellen, TX) for each of these proteins suggested that they are not required for evoked neurotransmitter release. HRS instead appears to function primarily in the endocytic pathway. It seems likely that most of the key proteins required for exocytosis are already known, and that several others currently under investigation may turn out not to be as important as originally thought.

\section{The pores}

One of the most significant achievements of cell membrane capacitance measurements has been their ability to track the kinetics of the activities of the fusion pore, the water channel that connects the vesicle interior with the extracellular space during exocytosis, and through which neurotransmitters are expelled out of the cell. The nature of the fusion pore, i.e. whether protein (Almers, 1990) or lipid (Monck and Fernandez, 1992), was the subject of much controversy in the early nineties, but was never fully established. Perhaps the recent identification of the many proteins involved at different stages of the exocytic process will help to bring an understanding of the composition of the pore closer. The overall picture is now much more complete, and it may not be as critical to know the exact nature of the exocytotic fusion pore as to assign a clear function to the known proteins at different stages of fusion pore formation and expansion. A key idea that has emerged over the last few years is whether and how the fusion pore maintains its structure in order to prevent full merging of vesicle and plasma membranes during exocytosis. The focus of experiments in recent years has therefore shifted toward identifying roles of particular proteins in the formation, maintenance and dispersal of the fusion pore. Technology employing fluorescence markers of exo- and endocytosis to track synaptic vesicles has brought new insight to our understanding of the mechanisms involved in fusion and in vesicle retrieval.

Manfred Lindau (Ithaca, NY) presented data on how fusion pores form in different secretory systems, and how these pores can be studied systematically using the patch-clamp technique. He presented a novel approach in which exocytotic fusion and fusion pore kinetics can be monitored and controlled in a calcium-dependent way in cell-free, inside-out patches from bovine chromaffin cells. This system might prove to be a fully accessible assay for calcium-dependent exocytosis. Wolf Almers (Portland, OR) presented data using evanescence wave microscopy applied to the giant synaptic terminal of goldfish bipolar retinal cells. He addressed the question of how vesicles reach their targets at the active zone (presumably the ribbon synapse), and at non-synaptic sites of the plasma membrane. In addition, he discussed measurements from the use of the dye FM1-43. FM dyes become incorporated from the aqueous medium into the lipid bilayer of vesicles during cycles of exo- and endocytosis. After removal of dye from the external medium, the dye within vesicles can potentially diffuse laterally into the plasma membrane and then into the medium following exocytosis. Almers' data suggested that the FM1-43 marker becomes rapidly dispersed through the plasma membrane by the full incorporation of the vesicle membrane but the technique might not detect transient fusion events. This is the first measurement of the rate of merging of these membranes, and it suggests extremely rapid expansion of the fusion pore at a synapse.

The above approach stops at the merging of the vesicle and plasma membranes, and does not explain how and when the vesicle membrane is recycled. To address this point, Richard Tsien, Jason Pyle and colleagues (Stanford, CA) described experiments using two fluorescence markers, FM1-43 and FM2-10 (Klingauf et al., 1998). Of the two, FM2-10 is known to dissociate from the membrane much more rapidly. The finding of the investigators, that the two dyes are differentially retained in vesicles, is consistent with the vesicle fusion pore being open for only a limited time. They identified conditions in which a significant percentage of synaptic vesicles (25\%) lose their FM2-10 while retaining some FM1-43, presumably due to only transient fusion. These vesicles retain their capacity to undergo rapid exocytosis. This is a clear demonstration that synaptic vesicles are very rapidly endocytosed (in less than a second) and then re-used, clearly pointing to a so called 'kiss-and-run' mechanism (Fesce et al., 1994) of synaptic vesicle exocytosis. They suggested that the pool of rapidly re-used synaptic vesicles could 


\section{meeting report}

be very effective during intermittent bursts of activity, preventing exhaustion of the rapidly releasable pool.

Further evidence that rapid endocytosis (on a timescale of a few seconds) might be important for rapid recycling came from data presented by Leon Lagnado (Cambridge, UK). He showed that calcium accumulation in retinal bipolar neurons accelerated rapid retrieval of vesicle membranes, and that the calcium chelator EGTA (even at $40 \mathrm{mM}$ ) does not prevent this fast endocytotic mechanism. It appears that, in certain neurons, a fast mechanism of endocytosis may play an important role in maintaining synaptic activity. Nevertheless, questions remain about the cellular processes and the molecules underlying this fast endocytosis.

The use of capacitance measurement provides a good method of monitoring single vesicle exocytosis since it records the net increase in cell surface area due to vesicle fusion. The simultaneous use of amperometry provides an independent measure of the exocytotic process, revealing the details of fusion pore formation, expansion and release of the vesicle contents. Guillermo Alvarez de Toledo, Lucia Tabares (Seville, Spain) and Manfred Lindau used patch amperometry recordings in chromaffin cells to show that chromaffin granules undergoing exocytosis are retrieved by a kiss-and-run mechanism when extracellular calcium is elevated (Ales et al., 1999). Using cell attached patch-clamp capacitance measurements, they showed that the fusion pore re-closes in a calcium-dependent manner after initially leaking catecholamine during the expansion of the fusion pore, and causing a bulk release once the fusion pore has opened. The overall picture that has developed is that the fusion pore initially expands slowly producing a long-lasting fusion event, and later closes back again to generate an 'empty granule' inside the cell. These types of event release most, if not all, of the vesicular contents and are different from transient fusion events (flicker) previously described in mast cells and chromaffin cells in which only partial release occurs at physiological levels of extracellular calcium. These recordings may provide a mechanistic model of how kiss-and-run could act at a synapse. However, exocytosis in synapses is considerably faster than in other cell types and, since neurotransmission is so important for neuronal function, more experiments are required to determine the details of the mechanism of exocytotic fusion pore function in central nervous system synapses.

Data discussed at this meeting point to the fact that the opening and closing of the fusion pore may be carefully regulated during the fusion process. One potential regulatory mechanism may be mediated via protein kinase C (PKC). Lindau had shown that activation of PKC increases the rate of fusion pore expansion during granule exocytosis in horse eosinophils (Scepek et al., 1998). Amperometry data presented by Burgoyne suggested that PKC activation could increase the initial rate of catecholamine release from individual chromaffin granules, possibly by an effect on fusion pore expansion (Graham et al., 2000). Independent evidence for a switch to a kiss-and-run type mechanism of exocytosis following PKC activation has recently come from the use of FM dyes in synaptosomes (Cousin and Robinson, 2000).

The end of the vesicle cycle story came from laboratories working on endocytosis. Harvey McMahon (Cambridge, UK) presented data suggesting that dynamin may act in a GTPdependent manner to pop vesicles off the plasma membrane. It kiss-and-run

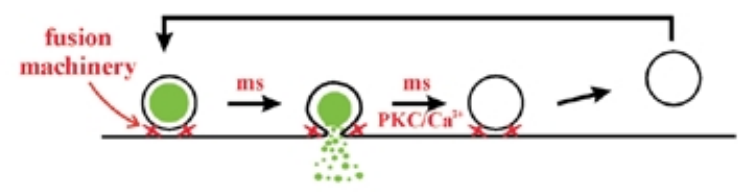

full fusion

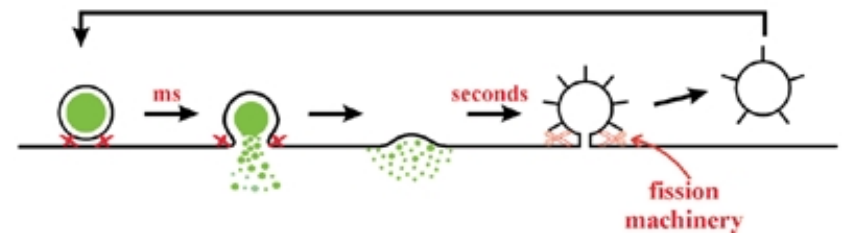

Fig. 1. Two extreme models for vesicle exo-/endocytosis are shown, with emphasis on the potential differences between 'kiss-and-run' and 'full fusion' mechanisms during the vesicle cycle. It may well be that the mechanisms are more similar than we have shown. It is also possible that what is designated fast endocytosis based on electrophysiological measurements is an intermediate form in which full fusion actually occurs, but is compensated for by rapid endocytosis via a clathrin-independent mechanism.

is clear that dynamin participates in clathrin-mediated endocytosis, but more conclusive evidence will be required to address the involvement of dynamin in rapid endocytosis of secretory vesicles. Wieland Huttner (Heidelberg, Germany) presented data showing that endophilin might be involved in site-specific endocytosis. He revealed that cholesterol might be actively involved in the process, and may act by constraining the patch of membrane to be retrieved by endocytosis. These results point to the idea that the formation of rafts composed of pure lipid and restrained by proteins of the membrane might be a crucial step for the selective endocytosis of membrane within synapses.

\section{Conclusions}

Agreement is growing regarding the identities of the key proteins that form the core of the fusion machinery. Considerable work is still needed, however, to sort out finally how these and other less well studied proteins act in concert to bring about membrane fusion. It is also clear that the evidence for rapidly reversible fusion events (kiss-and-run exocytosis) is growing and will be a focus of future work aimed at understanding the mechanics and regulation of this process. In Figure 1, we show two extreme models for vesicle exo-/endocytosis. In the first, kiss-and-run fusion is due to reversible use of the fusion machinery, allowing pore closure on a millisecond timescale. We also indicate that this results in total depletion of transmitter from the vesicle. However, it could also result in partial release from the vesicle if the fusion pore opens very briefly. The second model shows full fusion, with vesicle membrane retrieval via clathrin-coating and a fission machinery (dynamin) over a time course that could range from seconds to minutes. It is clear that a number of questions remain to be addressed in relation to these models. Is the fusion 


\section{meeting report}

machinery (the SNARE complex?) able to act in a reversible manner to allow rapid pore closure? Can kiss-and-run, under the influence of $\mathrm{Ca}^{2+}$ or PKC signaling, control the amount of neurotransmitter release per vesicle? What determines the usage of each pathway for vesicle exo-/endocytosis? Are these really distinct processes and could a dynamin-based fission machinery act on a sub-second or millisecond time scale? Further experimental manipulation of the protein components of the fusion and fission machinery, and examination of their exact roles during exo-/ endocytosis using high-resolution recording techniques, will help to resolve these questions and, hopefully, bring our understanding of proteins and pores closer together.

\section{References}

Ales, E., Tabares, L., Poyato, J.M., Valero, V., Lindau, M. and Alvarez de Toledo, G. (1999) High calcium concentrations shift the mode of exocytosis to the kiss-and-run mechanism. Nature Cell Biol., 1, 40-44.

Almers, W. (1990) Exocytosis. Annu. Rev. Physiol., 52, 607-624.

Cousin, M.A. and Robinson, P.J. (2000) Two mechanisms of synaptic vesicle recycling in rat brain nerve terminals. J. Neurochem., in press.

Fesce, R., Grohovaz, F., Valtorta, F. and Meldolesi, J. (1994) Neurotransmitter release: fusion or 'kiss-and-run'. Trends Cell Biol., 4, 1-4.

Graham, M.E., Fisher, R.J. and Burgoyne, R.D. (2000) Measurement of exocytosis by amperometry in adrenal chromaffin cells: effects of clostridial neurotoxins and activation of protein kinase $\mathrm{C}$ on fusion pore kinetics. Biochimie, 82, 469-479.

Klingauf, J., Kavalali, E.T. and Tsien, R.W. (1998) Kinetics and regulation of fast endocytosis at hippocampal synapses. Nature, 394, 581-585.

Monck, J.R. and Fernandez, J.M. (1992) The exocytotic fusion pore. J. Cell Biol., 119, 1395-1404.

Scales, S.J., Chen, Y.A., Yoo, B.Y., Patel, S.M., Doung, Y.-C. and Scheller, R.H. (2000) SNAREs contribute to the specificity of membrane fusion. Neuron, 26, 457-464.
Scepek, S., Coorssen, J.R. and Lindau, M. (1998) Fusion pore expansion in horse eosinophils is modulated by $\mathrm{Ca}^{2+}$ and protein kinase $\mathrm{C}$ via distinct mechanisms. EMBO J., 17, 4340-4345.

Sutton, R.B., Fasshauer, D., Jahn, R. and Brunger, A.T. (1998) Crystal structure of a SNARE complex involved in synaptic exocytosis at $2.4 \AA$ resolution. Nature, 395, 347-353.

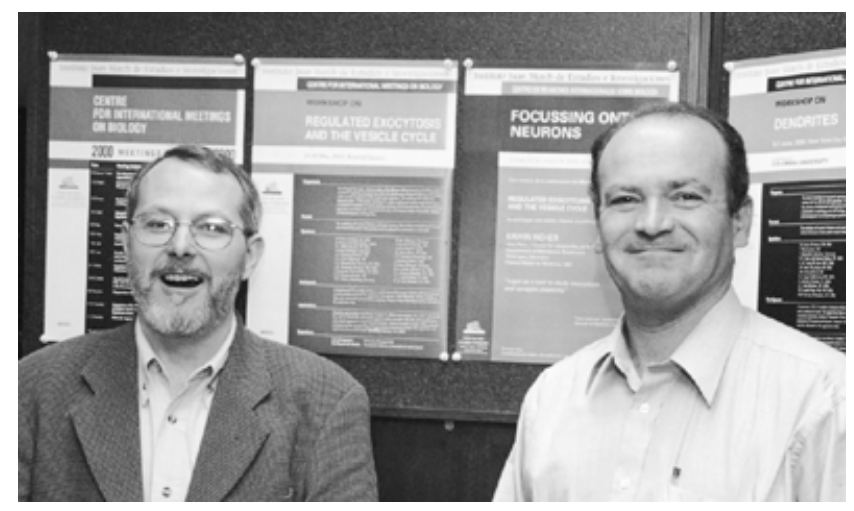

\section{Robert D. Burgoyne ${ }^{+}$and Guillermo Alvarez de Toledol}

The Physiological Laboratory, University of Liverpool, Crown Street, Liverpool L69 3BX, UK and ${ }^{1}$ Departamento de Fisiologia Medica y Biofisica, Facultad de Medicina, Universidad de Sevilla, 41009 Sevilla, Spain

${ }^{+}$Corresponding author. Tel: +44 151794 5305; Fax: +44 151794 5337; E-mail: burgoyne@liv.ac.uk

DOI: 10.1093/embo-reports/kvd074 